\title{
Desempenho de genótipos de bananeiras em cultivos irrigado e sequeiro, na região Centro Oeste Paulista
}

\author{
Adriana Novais Martins ${ }^{1}$, Nobuyoshi Narita ${ }^{2}$, Eduardo Suguino ${ }^{3}$, William Hiroshi S. Takata ${ }^{4}$ \\ ${ }^{1}$ Polo Regional Centro Oeste/APTA, UPD Marília, Marília, SP. ${ }^{2}$ Polo Regional da Alta Sorocabana/APTA, Presidente \\ Prudente, SP. ${ }^{3}$ Centro Avançado de Pesquisa de Cana/IAC, Campinas, SP. ${ }^{4}$ Universidade do Oeste Paulista - UNOESTE. E- \\ mail: adrianamartins@apta.sp.gov.br
}

\section{Resumo}

A bananeira (Musa sp.) é uma frutífera tropical, cultivada comercialmente em todo território brasileiro. Característica de regiões litorâneas, a banana vem ganhando espaço no Planalto Paulista, principalmente na região Centro Oeste do estado. Diante disso, o objetivo desse trabalho foi avaliar o desempenho de genótipos de bananeiras cultivadas sob irrigação por microaspersão e sequeiro nas condições edafoclimáticas do Centro Oeste Paulista. O plantio foi realizado em dezembro de 2015, no município de Vera Cruz -SP, utilizando mudas micropropagadas e aclimatadas. O delineamento experimental adotado foi de blocos ao acaso, com 6 tratamentos, em arranjo fatorial $3 \times 2$, sendo 3 genótipos e 2 ambientes de cultivo (irrigado e sequeiro). Os genótipos avaliados foram: BRS Princesa (subgrupo Maçã), Galil 18 (subgrupo Prata) e Grande Naine (subgrupo Cavendish). Foram avaliadas variáveis vegetativas (altura de planta, circunferência do pseudocaule e número de folhas vivas no florescimento), de produção (massa dos cachos e dos engaços, produtividade, número de pencas por cacho e número de frutos por penca) e caracterização física dos frutos (diâmetro, espessura de casca e comprimento). Conclui-se que a irrigação localizada por microaspersão interfere positivamente no desempenho dos genótipos BRS Princesa (subgrupo Maçã), Galil 18 (subgrupo Prata) e Grande Naine (subgrupo Cavendish), no município de Vera Cruz, na região Centro Oeste Paulista.

Palavras-chave: Musa sp.; bananicultura; fruticultura tropical.

Performance of banana genotypes in irrigated and non irrigated environments in the Central West region of São Paulo, Brazil

\begin{abstract}
The banana tree is a tropical fruit plant, grown commercially throughout Brazil. It's commonly found of coastal regions, the banana has been gaining space in the Paulista Plateau, mainly in the midwest region of the state. The objective of this work was to evaluate the performance of banana genotypes cultivated under microaspersion irrigation and rainfed in the edaphoclimatic conditions of Vera Cruz municipality in São Paulo state. The planting was carried out in December 2015, with micropropagated and acclimated seedlings. The statistical design adopted was randomized blocks, with 6 treatments, in a $3 \times 2$ factorial arrangement with 3 genotypes and 2 cultivation environments (irrigated and non irrigated). The genotypes evaluated were: BRS Princesa (Maçã subgroup), Galil 18 (Prata subgroup) and Grande Naine (Cavendish subgroup). Vegetative variables (plant height, pseudostem circumference and the number of live leaves in flowering); production variables (clusters and weight mass, productivity, number of hands per bunches and number of fruit for hand) and fruit quality (diameter, shell thickness and length), were evaluated. It is concluded that the localized irrigation by microaspersion positively interfered in the plant performance, improving the vegetative and productive characteristics of the BRS Princesa (Maçã subgroup), Galil 18 (Prata subgroup) and Grande Naine (Cavendish subgroup) genotypes, in Vera Cruz, midwest region of São Paulo state.
\end{abstract}

Keywords: Musa sp.; banana plantation; tropical fruticulture. 


\section{Introdução}

A bananeira é uma espécie frutífera tropical, originária da Ásia, cultivada em todo território nacional. O Brasil produziu em 2018, cerca de 6,8 milhões de toneladas da fruta, ocupando o quarto lugar no ranking mundial. São Paulo é o principal estado produtor de banana. Apesar da bananicultura comercial estar concentrada na faixa litorânea do estado, o Planalto Paulista vem aumentando significativamente o seu potencial produtivo (AGRIANUAL, 2019).

Nogueira et al. (2018) avaliaram dezenove genótipos de bananeiras em Rio Branco, Acre, cultivados sem irrigação suplementar. Foram analisadas características agronômicas durante três ciclos de produção, sendo que os genótipos Thap Maeo, FHIA 02, FHIA 18 e Maravilha foram os que mostraram melhores resultados, podendo ser incluídos nas recomendações para a bananicultura do estado do Acre.

Em experimentos conduzidos em Minas Gerais, Pereira et al. (2019) avaliaram genótipos de bananeiras originários da cultivar Prata-Anã, em sistema irrigado, no município de Bambuí, e em sequeiro no município de ljaci, entre 2015 e 2017. Os autores concluíram que a irrigação localizada aumentou a produção em cerca de $60 \%$, confirmando a viabilidade da suplementação hídrica em bananais comerciais.

Marques et al. (2011) estudaram genótipos de bananeiras cultivados em diferentes sistemas de irrigação nas condições semi-áridas, em Guanambi, Bahia. Os sistemas de irrigação por aspersão e microaspersão mostraram-se mais eficientes quando comparados ao sistema por gotejamento. Segundo os autores, essa superioridade pode ser atribuída à maior área molhada pelos sistemas de aspersão, que proporciona a melhor distribuição do sistema radicular das bananeiras, aumentando o vigor das plantas, concordando com os resultados obtidos por Donato et al. (2010).

No Planalto Paulista, a irrigação é uma técnica fundamental para a competitividade da bananicultura, pois esta região tem como característica climática, déficit hídrico acentuado durante o inverno (TEIXEIRA, 2008). Além disso, a irrigação favorece maior eficiência das adubações, principalmente a potássica, contribuindo significativamente para uma melhoria na nutrição das plantas (MARTINS et al., 2011).
O objetivo deste trabalho foi avaliar a irrigação localizada por microaspersão nas características vegetativas e produtivas de três genótipos de bananeiras, cultivados nas condições edafoclimáticas da região centro oeste do estado de São Paulo.

\section{Materiais e Métodos}

O experimento foi implantado em Argissolo eutrófico abrupto textura arenosa/média $\mathrm{A}$ moderado. A área de cultivo localiza-se no Sítio Bela Vista, município de Vera Cruz, estado de São Paulo $\left(22^{\circ} 14^{\prime} 04^{\prime \prime} S\right.$, $49^{\circ} 50^{\prime} 49^{\prime \prime} \mathrm{W}$, altitude de $\left.630 \mathrm{~m}\right)$. O clima predominante é o Cwa, segundo a classificação de Köppen, caracterizado por inverno seco (temperaturas inferiores a $18^{\circ} \mathrm{C}$ ) e verão quente (temperatura superior a $22^{\circ} \mathrm{C}$ ).

O plantio foi realizado em dezembro de 2015 , no espaçamento de $2,5 \times 3,0 \mathrm{~m}$, com mudas micropropagadas, e aclimatadas na Unidade de Pesquisa e Desenvolvimento de Marília (UPD Marília), Agência Paulista de Tecnologia dos Agronegócios - APTA, Secretaria de Agricultura e Abastecimento do Estado de São Paulo. A implantação e o manejo da cultura seguiram as recomendações técnicas para a região, de acordo com Teixeira et al. (1997). As adubações foram realizadas tendo como base resultados de análises de solo, sendo iguais tanto no sistema irrigado como no sequeiro. A área total do experimento foi de $2.250 \mathrm{~m}^{2}$, considerando-se as bordaduras laterais, sendo metade irrigada e a outra metade, sequeiro.

A irrigação foi realizada por meio do sistema de microaspersão, instalado entre as plantas, na linha de plantio, sendo 1 microaspersor (vazão de $45 \mathrm{~L} / \mathrm{h}$ ) para cada 2 bananeiras. A lâmina de água aplicada foi determinada por meio de 3 tensiômetros posicionados nas profundidades de 20,40 e 60 $\mathrm{cm}$, na área irrigada, e a leitura diária. $\mathrm{O}$ manejo da irrigação foi realizada de acordo com Borges et al. (2011a) e Coelho (2012), sendo considerados os valores obtidos pela curva de retenção de água pelo solo local, de modo a manter a disponibilidade hídrica adequada à cultura em todas as fases fenológicas das plantas, levando-se em consideração as condições climáticas predominantes durante o período de avaliação (Figura 1). 
Figura 1. Dados climáticos da região de Vera Cruz, SP. Temperaturas médias máxima e mínima $\left({ }^{\circ} \mathrm{C}\right)$ e chuva (mm). (Fonte: www.ciiagro.sp.gov.br)

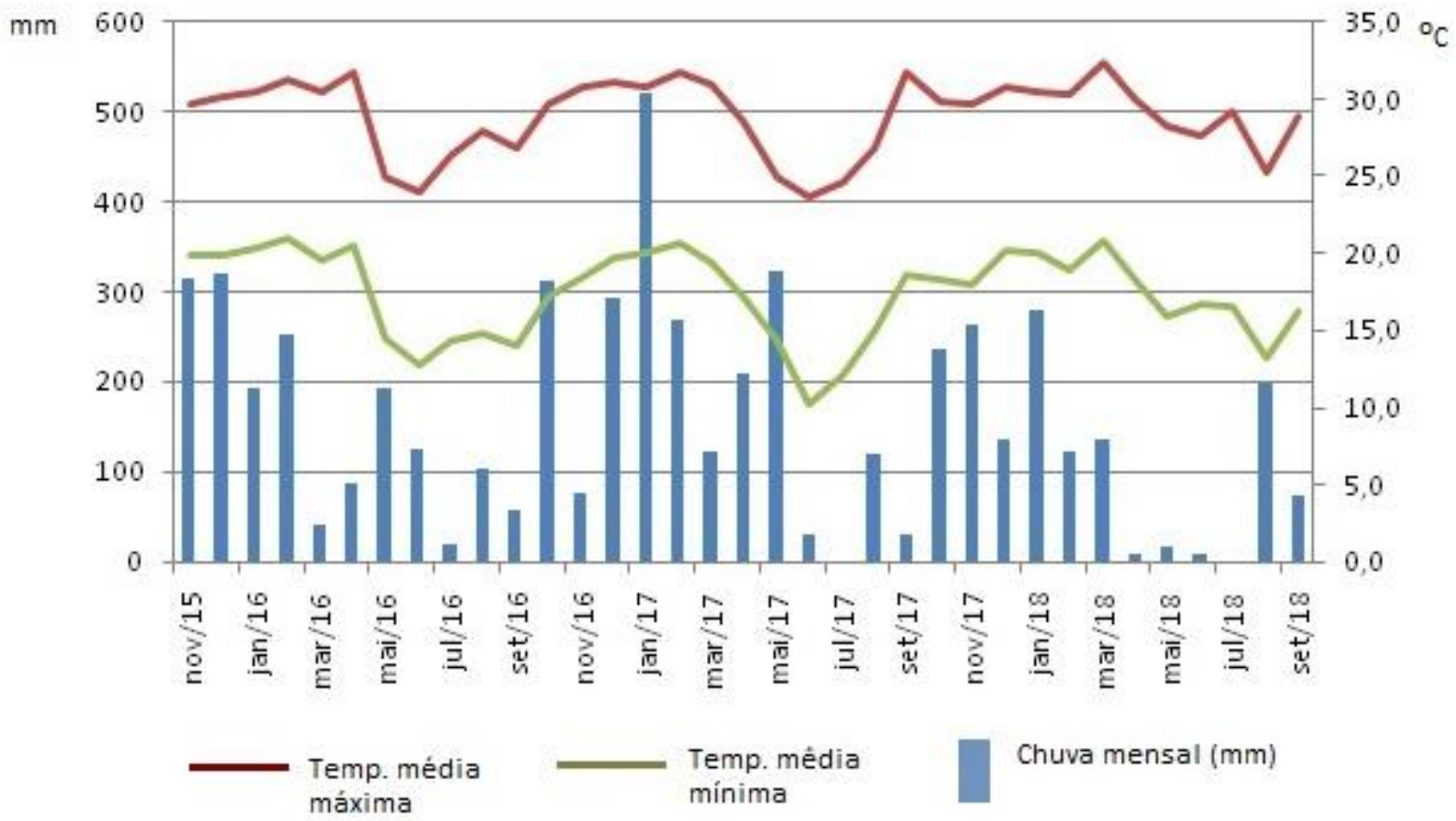

O delineamento experimental utilizado foi de blocos ao acaso, com 6 tratamentos, em arranjo fatorial $3 \times 2$, sendo 3 genótipos e 2 ambientes de cultivo (irrigado e sequeiro). Cada tratamento foi representado por 3 repetições de 12 plantas cada, totalizando 36 plantas por tratamento.

Os genótipos avaliados foram:

- BRS Princesa: híbrido tetraplóide AAAB, pertence ao subgrupo Maçã, resistente ao mal do Panamá (Fusarium oxysporum f.sp. cubense), Sigatoka Amarela (Mycosphaerella musicola) e Sigatoka Negra (Mycosphaerella fijiensis) (NOMURA et al., 2016).

- Galil 18: híbrido tetraplóide AAAB de Prata-Anã (subgrupo Prata), resistente à sigatoka-negra, moderadamente suscetível à sigatoka-amarela e tolerante ao Mal-do-Panamá (COSTA et al., 2012). -Grande Naine: grupo genômico AAA, subgrupo Cavendish, suscetível tanto à Sigatoka Amarela, como a Negra (ALVES et al., 2010)

As avaliações foram realizadas em 3 ciclos produtivos. Na época do florescimento foram avaliadas: altura da planta, do nível do solo à inserção da inflorescência (AP, cm), circunferência do pseudocaule a $20 \mathrm{~cm}$ do solo $(C P, \mathrm{~cm})$ e número de folhas vivas (NFV). Durante a colheita, foram avaliados: massa do cacho (MC, $\mathrm{kg}$ ); massa do engaço ( $\mathrm{ME}, \mathrm{kg}$ ), número de pencas por cacho (NPC), número de frutos por penca (NFP); comprimento do fruto $(\mathrm{CF}, \mathrm{cm})$, média dos 4 frutos centrais da penca número 2 de cada cacho colhido, utilizando-se fita métrica; diâmetro do fruto $(D F, c m)$, média dos frutos utilizados para avaliação do comprimento, utilizando-se paquímetro digital e espessura de casca (EC, $\mathrm{mm}$ ), avaliada nos mesmos frutos utilizados para comprimento e diâmetro, cortando-os ao meio e medindo a espessura de casca com paquímetro digital . Por fim, calculou-se a produtividade (PROD, $\mathrm{t} \mathrm{ha}^{-1}$ ), considerando-se a massa do cacho, descontando-se a massa do engaço, e o espaçamento de plantio adotado na área experimental.

Os dados foram submetidos à análise de variância e ao constatar significância pelo teste $F$, as médias foram comparadas pelo Teste de Tukey, a $5 \%$ de probabilidade. Os resultados obtidos foram analisados, pelo software SISVAR 5.6 (FERREIRA, 2014).

\section{Resultados e Discussão}

Com relação à altura das plantas (Tabela 1) não foram encontradas diferenças significativas entre as bananeiras irrigadas e sequeiro. Foi observada diferença estatística significativa entre os genótipos, visto que são materiais genéticos pertencentes a grupos genômicos diferentes. O genótipo BRS Princesa alcançou os maiores valores, com plantas ultrapassando 3 metros de altura. De acordo com Faria et al. (2010), essa variável é um descritor importante no ponto de 
vista fitotécnico e de melhoramento, pois interfere diretamente no manejo da cultura; entretanto apresenta elevada variabilidade, sendo afetada pelos tratos culturais adotados no campo de avaliação.

Tabela 1. Altura da planta (AP, cm), circunferência do pseudocaule (CP, cm) e número de folhas vivas (NFV), avaliados no florescimento das bananeiras em 3 ciclos de cultivo, sob condições irrigadas e sequeiro. Vera Cruz, São Paulo.

\begin{tabular}{|c|c|c|c|c|c|c|c|}
\hline \multirow{2}{*}{ Variável } & \multirow{2}{*}{ Genótipo } & \multicolumn{2}{|c|}{ CICLO I } & \multicolumn{2}{|c|}{ CICLO II } & \multicolumn{2}{|c|}{ CICLO III } \\
\hline & & IRRIG. & SEQ. & IRRIG. & SEQ. & IRRIG. & SEQ. \\
\hline \multirow{3}{*}{$\mathrm{AP}(\mathrm{cm})$} & BRS Princesa & $163,7 \mathrm{aA}$ & $153,1 \mathrm{aA}$ & $268,4 \mathrm{aA}$ & $279,2 \mathrm{aA}$ & $319,9 \mathrm{aA}$ & 325,8 aA \\
\hline & Galil 18 & $118,9 \mathrm{bA}$ & $110,0 \mathrm{bA}$ & $202,7 \mathrm{bA}$ & $196,0 \mathrm{bA}$ & $238,9 \mathrm{bA}$ & $232,6 \mathrm{bA}$ \\
\hline & Grande Naine & $95,3 \mathrm{cA}$ & $93,7 \mathrm{cA}$ & $181,6 \mathrm{cA}$ & $166,9 \mathrm{cA}$ & $211,9 \mathrm{cA}$ & $206,2 \mathrm{cA}$ \\
\hline \multicolumn{2}{|c|}{ Média geral } & 125,9 & 118,9 & 217,6 & 214,0 & 256,9 & 254,9 \\
\hline \multicolumn{2}{|c|}{ C.V. (\%) } & \multicolumn{2}{|c|}{5,19} & \multicolumn{2}{|c|}{3,91} & \multicolumn{2}{|c|}{2,07} \\
\hline & BRS Princesa & 44,6 aA & $45,5 \mathrm{aA}$ & $56,9 \mathrm{aA}$ & $61,1 \mathrm{aA}$ & $64,1 \mathrm{bA}$ & $61,3 \mathrm{bB}$ \\
\hline \multirow{2}{*}{$\mathrm{CP}(\mathrm{cm})$} & Galil 18 & $42,7 \mathrm{aA}$ & $43,1 \mathrm{aA}$ & $56,2 \mathrm{aA}$ & 55,9 aA & 70,3 aA & 69,6 aA \\
\hline & Grande Naine & $39,6 \mathrm{aA}$ & 41,6 aA & 52,4 aA & $54,2 \mathrm{aA}$ & 65,1 bA & $61,1 \mathrm{bB}$ \\
\hline \multicolumn{2}{|c|}{ Média geral } & 42,3 & 43,4 & 55,2 & 57,1 & 66,5 & 64,0 \\
\hline \multicolumn{2}{|c|}{ C.V. (\%) } & \multicolumn{2}{|c|}{4,95} & \multicolumn{2}{|c|}{4,43} & \multicolumn{2}{|c|}{4,62} \\
\hline \multirow{3}{*}{ NFV } & BRS Princesa & $8,2 \mathrm{aA}$ & $7,1 \mathrm{aB}$ & $9,9 \mathrm{aA}$ & 9,3 aA & $8,6 \mathrm{aA}$ & $7,2 \mathrm{aB}$ \\
\hline & Galil 18 & $8,2 \mathrm{aA}$ & $7,0 \mathrm{aB}$ & 8,9 aA & 8,6 aA & $6,6 \mathrm{bA}$ & $6,2 \mathrm{aA}$ \\
\hline & Grande Naine & $5,8 \mathrm{bA}$ & $4,1 \mathrm{bB}$ & $9,8 \mathrm{aA}$ & 9,0 aA & $5,4 \mathrm{bA}$ & $4,7 \mathrm{bA}$ \\
\hline \multicolumn{2}{|c|}{ Média geral } & 7,4 & 6,1 & 9,5 & 8,9 & 6,9 & 6,0 \\
\hline \multicolumn{2}{|c|}{ C.V. (\%) } & \multicolumn{2}{|c|}{5,92} & \multicolumn{2}{|c|}{7,02} & \multicolumn{2}{|c|}{8,66} \\
\hline
\end{tabular}

* letras iguais minúsculas na coluna e maiúscula na linha, dentro de cada ciclo produtivo, não diferem entre si pelo teste de Tukey, a 5\% de probabilidade.

Não foram identificadas diferenças na circunferência dos pseudocaules nos dois primeiros ciclos, entre os genótipos e entre os sistemas hídricos (Tabela 1). Entretanto, na avaliação do terceiro ciclo de produção, as plantas mostraram diferenças entre os genótipos, sendo que a Galil 18 (tipo Prata) retornou valores maiores de circunferência, com plantas mais robustas que os outros dois genótipos, mas inferiores aos encontrados por Marques et al. (2011), em experimento conduzido na Bahia, provavelmente devido às características distintas de solo, tanto físicas quanto químicas. Os genótipos BRS Princesa (tipo Maçã) e Grande Naine (subgrupo Cavendish) apresentaram maiores valores, para esta variável, nas plantas irrigadas, devido ao melhor aproveitamento dos nutrientes no ambiente irrigado, concordando com os resultados obtidos por Martins et al. (2011), Villas Boas et al. (2005) e Havlin et al. (2005).

Com relação ao número de folhas vivas no florescimento, observam-se diferenças entre os genótipos no primeiro e no terceiro ciclos de produção, com redução da variável para o genótipo Grande Naine. Isso ocorreu uma vez que este material é suscetível às doenças foliares (Sigatokas Amarela e Negra), apresentando maior dano devido á maior incidência de Sigatoka Amarela nos ciclos I (2016) e III (2018). De acordo com a Figura 1, observa-se que a ocorrência de temperaturas mais elevadas, associadas a chuvas, nos invernos de 2016 e 2018 favoreceu o desenvolvimento do fungo, ocasionando maior incidência da doença nos bananais da região. Além dos desequilíbrios nutricionais, principalmente entre $\mathrm{N}$ e $\mathrm{K}$, que aceleram o processo de senescência foliar, diminuindo o número de folhas fotossinteticamente ativas nas plantas (TEIXEIRA et al., 2001).

Em termos de massa dos cachos colhidos ocorreram diferenças significativas entre os genótipos (Tabela 2). Porém, é importante ressaltar os maiores valores obtidos no sistema irrigado em todos os ciclos e para todos os genótipos, evidenciando uma ação positiva da suplementação hídrica para a bananicultura local. Os resultados obtidos neste trabalho concordam 
com os divulgados por Martins et al. (2011) em experimento também conduzido no Planalto Paulista, mas instalados em solos mais argilosos, comparando o sistema de manejo em sequeiro com diversas lâminas de água aplicadas por microaspersão em bananeiras da variedade Willians (subgrupo Cavendish).

Tabela 2. Massa do cacho ( $M C, \mathrm{~kg})$, massa do engaço $(\mathrm{ME}, \mathrm{kg})$ e produtividade ( $\left.\mathrm{PROD}, \mathrm{t} \mathrm{ha}{ }^{-1}\right)$ das bananeiras em 3 ciclos de cultivo, sob condições irrigadas e sequeiro. Vera Cruz, São Paulo.

\begin{tabular}{|c|c|c|c|c|c|c|c|}
\hline \multirow{2}{*}{ Variável } & \multirow{2}{*}{ Genótipo } & \multicolumn{2}{|c|}{ CICLO I } & \multicolumn{2}{|c|}{ CICLO II } & \multicolumn{2}{|c|}{ CICLO III } \\
\hline & & IRRIG. & SEQ. & IRRIG. & SEQ. & IRRIG. & SEQ. \\
\hline \multirow{3}{*}{$\mathrm{MC}(\mathrm{kg})$} & BRS Princesa & $10,9 \mathrm{cA}$ & $9,6 \mathrm{cB}$ & $14,0 \mathrm{cA}$ & $11,3 \mathrm{cB}$ & $10,8 \mathrm{bA}$ & $8,3 \mathrm{cB}$ \\
\hline & Galil 18 & $14,7 \mathrm{bA}$ & $12,3 \mathrm{bB}$ & $16,9 \mathrm{bA}$ & $13,9 \mathrm{bB}$ & $12,0 \mathrm{bA}$ & $10,5 \mathrm{bB}$ \\
\hline & Grande Naine & 20,4 aA & $17,4 \mathrm{aB}$ & $30,1 \mathrm{aA}$ & $24,1 \mathrm{aB}$ & $20,5 \mathrm{aA}$ & $15,2 \mathrm{aB}$ \\
\hline \multicolumn{2}{|c|}{ Média geral } & 15,3 & 13,1 & 20,3 & 16,4 & 14,4 & 11,3 \\
\hline \multicolumn{2}{|c|}{ C.V. (\%) } & \multicolumn{2}{|c|}{8,97} & \multicolumn{2}{|c|}{8,08} & \multicolumn{2}{|c|}{7,22} \\
\hline & BRS Princesa & $1,06 \mathrm{bA}$ & $0,97 \mathrm{bA}$ & $1,33 \mathrm{bA}$ & $1,25 \mathrm{bA}$ & $1,20 \mathrm{aA}$ & $1,08 \mathrm{aA}$ \\
\hline \multirow{2}{*}{ ME (kg) } & Galil 18 & $1,15 \mathrm{bA}$ & $1,11 \mathrm{abA}$ & $1,50 \mathrm{abA}$ & 1,47 aA & $1,24 \mathrm{aA}$ & $1,08 \mathrm{aA}$ \\
\hline & Grande Naine & $1,27 \mathrm{aA}$ & 1,16 aA & $1,63 \mathrm{aA}$ & $1,62 \mathrm{aA}$ & $1,18 \mathrm{aA}$ & $1,10 \mathrm{aA}$ \\
\hline \multicolumn{2}{|c|}{ Média geral } & 1,16 & 1,08 & 1,49 & 1,45 & 1,21 & 1,09 \\
\hline \multicolumn{2}{|c|}{ C.V. (\%) } & \multicolumn{2}{|c|}{5,68} & \multicolumn{2}{|c|}{7,26} & \multicolumn{2}{|c|}{7,20} \\
\hline \multirow{3}{*}{$\begin{array}{c}\text { PROD** } \\
\left(\mathrm{t} \mathrm{ha}^{-1}\right)\end{array}$} & BRS Princesa & $13,1 \mathrm{cA}$ & $11,5 \mathrm{cB}$ & $16,9 \mathrm{cA}$ & $13,3 \mathrm{cB}$ & $12,8 \mathrm{bA}$ & $9,6 \mathrm{cB}$ \\
\hline & Galil 18 & $18,1 \mathrm{bA}$ & $15,0 \mathrm{bB}$ & $20,6 \mathrm{bA}$ & $16,7 \mathrm{bB}$ & $14,4 \mathrm{bA}$ & $12,5 \mathrm{bB}$ \\
\hline & Grande Naine & $25,5 \mathrm{aA}$ & $21,6 \mathrm{aB}$ & $38,0 \mathrm{aA}$ & $29,9 \mathrm{aB}$ & 25,8 aA & $18,8 \mathrm{aB}$ \\
\hline \multicolumn{2}{|c|}{ Média geral } & 18,9 & 16,0 & 25,2 & 19,9 & 17,7 & 13,6 \\
\hline \multicolumn{2}{|c|}{ C.V. (\%) } & \multicolumn{2}{|c|}{9,26} & \multicolumn{2}{|c|}{10,58} & \multicolumn{2}{|c|}{9,22} \\
\hline
\end{tabular}

* letras iguais minúsculas na coluna e maiúscula na linha, dentro de cada ciclo produtivo, não diferem entre si pelo teste de Tukey, a $5 \%$ de probabilidade.

** considerando o espaçamento adotado no experimento em questão.

A massa do engaço não diferiu entre os sistemas irrigado e sequeiro, mostrando diferença estatística somente entre os genótipos, nos dois primeiros ciclos (Tabela 2).

Considerando-se o espaçamento de plantio adotado no experimento, a produtividade média diferiu tanto para os genótipos, como para os sistemas de produção. A superioridade dos valores obtidos para o sistema irrigado evidencia os benefícios da irrigação para o cultivo de banana de qualquer subgrupo nas condições regionais, corroborando as afirmações realizadas por Teixeira (2008) e Martins et al. (2011), uma vez que a região Centro Oeste paulista apresenta déficit hídrico acentuado durante o inverno.

Com relação ao número de pencas por cacho (Tabela 3) observa-se uma nítida diferença entre os genótipos, corroborando com os resultados obtidos para massa dos cachos. A irrigação interferiu positivamente nesta variável nos 3 ciclos produtivos. Os valores médios do número de pencas por cacho foi inferior aos relatados em experimentos de Borges et al. (2011b), cujo experimento foi conduzido sem irrigação em Andirá, PR, e Martins et al. (2011), em trabalho realizado na cidade de Palmital, SP, utilizando sistemas irrigado e sequeiro. Esta variável deve ser mensurada em experimentos de bananicultura por se tratar da principal parte comercializada desta frutífera tropical. 
Tabela 3. Número de pencas por cacho (NPC), número de frutos por penca (NFP), diâmetro do fruto (DF, $\mathrm{cm})$, espessura de casca $(\mathrm{EC}, \mathrm{mm})$ e comprimento dos frutos $(\mathrm{CF}, \mathrm{cm})$ de 3 genótipos de bananeiras, em 3 ciclos de produção, em condições irrigadas e sequeiro. Vera Cruz, São Paulo.

\begin{tabular}{|c|c|c|c|c|c|c|c|}
\hline \multirow{2}{*}{ Variável } & \multirow{2}{*}{ Genótipo } & \multicolumn{2}{|c|}{ CICLO I } & \multicolumn{2}{|c|}{ CICLO II } & \multicolumn{2}{|c|}{ CICLO III } \\
\hline & & IRRIG. & SEQ. & IRRIG. & SEQ. & IRRIG. & SEQ. \\
\hline & BRS Princesa & $6,8 \mathrm{cA}$ & $6,2 \mathrm{cA}$ & $8,8 \mathrm{cA}$ & $6,9 \mathrm{cB}$ & $6,1 \mathrm{bA}$ & $5,2 \mathrm{bB}$ \\
\hline \multirow[t]{2}{*}{ NPC } & Galil 18 & $8,0 \mathrm{bA}$ & $7,7 \mathrm{bA}$ & $10,0 \mathrm{bA}$ & $8,9 \mathrm{bB}$ & $7,0 \mathrm{abA}$ & $6,2 \mathrm{abA}$ \\
\hline & Grande Naine & 9,5 aA & $8,4 \mathrm{aB}$ & $11,2 \mathrm{aA}$ & $9,8 \mathrm{aB}$ & 7,7 aA & $6,7 \mathrm{aB}$ \\
\hline \multicolumn{2}{|c|}{ Média geral } & 8,1 & 7,4 & 10,0 & 8,5 & 6,9 & 6,0 \\
\hline \multicolumn{2}{|c|}{ C.V. (\%) } & \multicolumn{2}{|c|}{5,76} & \multicolumn{2}{|c|}{6,79} & \multicolumn{2}{|c|}{7,34} \\
\hline & BRS Princesa & $10,9 \mathrm{bA}$ & $11,8 \mathrm{bA}$ & $15,1 \mathrm{bA}$ & $15,1 \mathrm{cA}$ & $13,0 \mathrm{bA}$ & $12,7 \mathrm{bB}$ \\
\hline \multirow{2}{*}{ NFP } & Galil 18 & 14,6 aA & $14,6 \mathrm{aA}$ & $18,4 \mathrm{aA}$ & $17,4 \mathrm{aB}$ & 15,8 aA & $14,4 \mathrm{aB}$ \\
\hline & Grande Naine & 13,9 aA & $13,8 \mathrm{aA}$ & $15,8 \mathrm{bA}$ & $16,4 \mathrm{bA}$ & 15,3 aA & $13,6 \mathrm{abB}$ \\
\hline \multicolumn{2}{|c|}{ Média geral } & 13,1 & 13,4 & 16,4 & 16,3 & 14,7 & 13,6 \\
\hline \multicolumn{2}{|c|}{ C.V. (\%) } & \multicolumn{2}{|c|}{8,43} & \multicolumn{2}{|c|}{5,50} & \multicolumn{2}{|c|}{7,79} \\
\hline & BRS Princesa & $3,08 \mathrm{bA}$ & $2,97 \mathrm{bA}$ & $3,26 \mathrm{bA}$ & $3,20 \mathrm{aA}$ & $3,11 \mathrm{aA}$ & $2,91 \mathrm{aB}$ \\
\hline \multirow[t]{2}{*}{$\mathrm{DF}(\mathrm{cm})$} & Galil 18 & $3,11 \mathrm{abA}$ & $3,09 a b A$ & $3,41 \mathrm{abA}$ & $3,20 \mathrm{aB}$ & 3,07 aA & $3,02 \mathrm{aA}$ \\
\hline & Grande Naine & $3,40 \mathrm{aA}$ & $3,34 \mathrm{aA}$ & $3,47 \mathrm{aA}$ & 3,37 aA & 3,18 aA & $2,92 \mathrm{aB}$ \\
\hline \multicolumn{2}{|c|}{ Média geral } & 3,20 & 3,13 & 3,38 & 3,26 & 3,12 & 2,95 \\
\hline \multicolumn{2}{|c|}{ C.V. (\%) } & \multicolumn{2}{|c|}{8,31} & \multicolumn{2}{|c|}{5,68} & \multicolumn{2}{|c|}{7,73} \\
\hline \multirow{3}{*}{$\mathrm{EC}(\mathrm{mm})$} & BRS Princesa & $5,39 \mathrm{cA}$ & $5,53 \mathrm{bA}$ & $5,58 \mathrm{cA}$ & $5,69 \mathrm{cA}$ & $5,51 \mathrm{cB}$ & $5,79 \mathrm{cA}$ \\
\hline & Galil 18 & $5,60 \mathrm{bA}$ & $5,69 \mathrm{bA}$ & $5,98 \mathrm{bB}$ & $6,15 \mathrm{bA}$ & $5,96 \mathrm{bA}$ & $6,20 \mathrm{bA}$ \\
\hline & Grande Naine & $6,37 \mathrm{aA}$ & $6,65 \mathrm{aA}$ & $6,52 \mathrm{aA}$ & $6,63 \mathrm{aA}$ & $6,56 \mathrm{aB}$ & 6,78 aA \\
\hline \multicolumn{2}{|c|}{ Média geral } & 5,79 & 5,96 & 6,03 & 6,16 & 6,01 & 6,26 \\
\hline \multicolumn{2}{|c|}{ C.V. (\%) } & \multicolumn{2}{|c|}{7,40} & \multicolumn{2}{|c|}{6,22} & \multicolumn{2}{|c|}{6,60} \\
\hline \multirow{3}{*}{$\mathrm{CF}(\mathrm{cm})$} & BRS Princesa & $11,62 \mathrm{bA}$ & $12,04 \mathrm{bA}$ & $12,78 \mathrm{cA}$ & $13,00 \mathrm{bA}$ & $12,01 \mathrm{bA}$ & $11,37 \mathrm{bA}$ \\
\hline & Galil 18 & $15,35 \mathrm{aA}$ & $15,32 \mathrm{aA}$ & $16,39 \mathrm{bA}$ & $16,23 \mathrm{aA}$ & $13,87 \mathrm{aA}$ & $13,05 \mathrm{aB}$ \\
\hline & Grande Naine & $16,32 \mathrm{aA}$ & 15,56 aA & $17,40 \mathrm{aA}$ & $16,13 \mathrm{aB}$ & $14,75 \mathrm{aA}$ & $13,74 \mathrm{aB}$ \\
\hline \multicolumn{2}{|c|}{ Média geral } & 14,43 & 14,31 & 15,52 & 15,12 & 13,54 & 12,72 \\
\hline \multicolumn{2}{|c|}{ C.V. (\%) } & & & & & & \\
\hline
\end{tabular}

* letras iguais minúsculas na coluna e maiúscula na linha, dentro de cada ciclo produtivo, não diferem entre si pelo teste de Tukey, a $5 \%$ de probabilidade.

A suplementação hídrica interferiu positivamente no número de frutos por penca, somente no ciclo III. De acordo com Holder e Gumbs (1982), deficiência hídrica na fase de florescimento, acarreta diminuição das flores femininas na diferenciação floral, promovendo redução do número de frutos. No ciclo II somente a 'Galil 18' apresentou diferença estatística nesse atributo. Houve diferença entre os genótipos em todos os ciclos produtivos (Tabela 3 ).

Em termos de caracterização dos frutos, os resultados evidenciam diferenças entre os genótipos. A irrigação beneficiou algumas das variáveis avaliadas, principalmente no ciclo III. O 'Galil 18' apresentou frutos com maior diâmetro e menor espessura de casca nas plantas irrigadas, no ciclo II, e maior comprimento de frutos no ciclo III. Já o 'BRS Princesa' mostrou ação benéfica da irrigação nestas variáveis apenas no ciclo III. O
'Grande Naine' também apresentou melhores resultados no ciclo III, nas plantas irrigadas, sendo que o comprimento dos frutos irrigados também foi maior no ciclo II. Diversos autores encontraram benefícios da irrigação na caracterização dos frutos de bananeiras (FARIA et al., 2010; MARQUES et al., 2011; MARTINS et al., 2011); portanto pode-se inferir que o suprimento hídrico adequado, principalmente nas fases fenológicas de florescimento e frutificação traga benefícios à produção e qualidade dos frutos formados, beneficiando o valor agregado destes.

\section{Conclusão}

A irrigação localizada por microaspersão interfere positivamente nas características vegetativas e produtivas das bananeiras dos genótipos BRS Princesa (subgrupo Maçã), Galil 18 
(subgrupo Prata) e Grande Naine (subgrupo Cavendish), na região Centro Oeste Paulista.

\section{Agradecimentos}

Os autores agradecem ao Fundo Estadual de Recursos Hídricos - FEHIDRO pelo apoio financeiro para a realização deste trabalho (contrato 469/2015, empreendimento 2015-AP697); à Associação dos Produtores Rurais, Artesãos e Produtos Alimentícios Caseiros de Vera Cruz - Associação da Feira da Lua, pela administração financeira do projeto, ao Sr. Paulo Roberto Medeiros da Silva, proprietário do Sítio Bela Vista, Vera Cruz, SP, pela cessão da área e colaboração para manutenção da área experimental e ao Sr. Ângelo Domingos Rossi, pela idealização e comprometimento com a fruticultura regional.

\section{Referências}

AGRIANUAL. Banana. São Paulo: FNP Consultoria \& Comércio, 2019. p. 152-160

ALVES, M.S.; COELHO, E.F.; PAZ, V.P.S.; ANDRADE NETO, T.M. Crescimento e produtividade da bananeira cv. Grande Naine sob diferentes combinações de nitrato de cálcio e ureia. Revista Ceres, Viçosa, v.57, n.1, p.125-131, 2010. http://dx.doi.org/10.1590/S0034-

\section{X2010000100020}

BORGES, A.L.; COELHO, E.F.; COSTA, E.L.; TEIXEIRA, A.H.C. Irrigação e fertirrigação na cultura da banana. In: SOUZA, V.F.; MAROUELLI, W.A.; COELHO, E.F.; PINTO, J.M.; COELHO FILHO, M.A. Irrigação e fertirrigação em fruteiras e hortaliças. Brasília: Embrapa Informação Tecnológica, 2011a. p. 369-397.

BORGES, R.S.; SILVA, S.O.; OLIVEIRA, F.T.; ROBERTO, S.R. Avaliação de genótipos de bananeira no Norte do estado do Paraná. Revista Brasileira de Fruticultura, Jaboticabal, v.33, n.1, p.291-296, 2011b.

http://dx.doi.org/10.1590/S0100$\underline{29452011005000034}$.

COELHO, E.F. Irrigação da bananeira. Brasília: EMBRAPA, 2012. 280 p.

COSTA, F.S.; COELHO, E.F.; BORGES, A.L.; PAMPONET, A.J.M.; SILVA, A.A.S.M.; AZEVEDO, N.F. Crescimento, produção e acúmulo de potássio em bananeira 'Galil 18' sob irrigação e fertilização potássica. Pesquisa Agropecuária Brasileira, Brasília, v.47, n.3, p.409-416, 2012. http://dx.doi.org/10.1590/S0100-

204X2012000300013

DONATO, S.L.R.; LÉDO, A.A.; PEREIRA, M.C.T.; COELHO, E.F.; COTRIM, C.E. Estado nutricional de bananeiras tipo Prata sob diferentes sistemas de irrigação. Pesquisa Agropecuária Brasileira, Brasília, v.45, n.9, p.980-988, 2010. http://dx.doi.org/10.1590/S0100-

204X2010000900007.

FARIA, H.C.; DONATO, S.L.R.; PEREIRA, N.C.T.; SILVA, S.O. Avaliação fitotécnica em bananeiras tipo Terra sob irrigação em condições semi-áridas. Ciência e Agrotecnologia, Lavras, v.34, n.4, p.830836, 2010. http://dx.doi.org/10.1590/S141370542010000400006

FERREIRA, D.F. Sisvar: a Guide for its Bootstrap procedures in multiple comparisons. Ciência \& Agrotecnologia, Lavras, v.38, n.2, p.109-112, $2014 . \quad$ http://dx.doi.org/10.1590/S141370542014000200001.

HAVLIN, J.L.; BEATON, J.D.; TISDALE, S.L.; NELSON, W.L. Soil fertility and fertilizers (7 th. ed) Upper Saddle River: Pearson Education, 2005. 515p.

HOLDER, G.D.; GUMBS, F.G. Effects of water supply during floral initiation and differentiation on female flower production by Robusta bananas. Experimental Agriculture, Cambridge, v.18, n.2, p.183-193, 1982. https://doi.org/10.1017/\$0014479700013661

MARQUES, P.R.R.; DONATO, S.L.R.; PEREIRA, M.C.T.; COELHO, E.F.; ARANTES, A.M. Características agronômicas de bananeiras tipo Prata sob diferentes sistemas de irrigação. Pesquisa Agropecuária Brasileira, Brasília, v.46, n.8, p.852-859, 2011. http://dx.doi.org/10.1590/s0100$\underline{204 \times 2011000800010}$

MARTINS, A.N.; TEIXEIRA, L.A.J.; SUGUINO, E.; HASHIMOTO, J.M.; NARITA, N. Irrigação e adubação potássica via fertirrigação em bananeira Willians - produção e qualidade de frutos. Revista Brasileira de Fruticultura, Jaboticabal, v.33, n.esp. 1, p.743-751, 2011. http://dx.doi.org/10.1590/S0100-

$\underline{29452011000500104}$ 
NOGUEIRA, S.R.; ANDRADE NETO, R.C.; CAPISTRANO, M.C.; LESSA, L.S.; ALÉCIO, M.R.; SANTOS, V.B. Performance of banana genotypes in Rio Branco, Acre, Brazil. Revista Brasileira de Ciências Agrárias, Recife, v.13, n.4, e. 5576, 2018. http://dx.doi.org/10.5039/agraria.v13i4a5576.

NOMURA, E.S.; CUQUEL, F.L.; DAMATTO JUNIOR, E.R.; FUZITANI, E.J.; BORGES, A.L.; SAES, L.A. Nitrogen and potassium fertilization on 'Caipira' and 'BRS Princesa' bananas in the Ribeira Valley. Revista Brasileira de Engenharia Agrícola e Ambiental, Campina Grande, v.20, n.8, p.702-708, 2016. http://dx.doi.org/10.1590/18071929/agriambi.v20n8p702-708

PEREIRA, L.V.; ANDRADE, J.C.; ALVARENGA, A.A.; MALTA, M.R.; NORBERTO, P.M.; LEITE, G.F.; SILVA, S.O. Productive behavior of cultivars and banana genotype originating from 'Prata-Anã', irrigated and non irrigated in the State of Minas Gerais, Brazil. International Journal of Environmental \& Agriculture Research, v.5, n.1, 2019. Disponível em:

https://ijoear.com/assets/articles menuscripts/fil e/IJOEAR-JAN-2019-1.pdf. Acesso em: 05 jun. 2019.

TEIXEIRA, L.A.J.; SPIRONELLO, A.; QUAGGIO, J.A.; FURLANI, P. Banana. In: RAIJ, B. van et al. (Ed.) Recomendações de adubação e calagem para o Estado de São Paulo. 2. ed. Campinas: IAC, 1997. p. 131-132. (Boletim Técnico, 100).

TEIXEIRA, L.A.J. Bananicultura no Planalto Paulista. In: LEONEL, S.; DAMATTO JUNIOR, E.; RUGGIERO, C.; SAMPAIO, A.C. (Org.) Workshop sobre inovações tecnológicas em bananicultura. Botucatu: FCA/UNESP, 2008. CD-ROM.

TEIXEIRA, L.A.J.; RUGGIERO, C.; NATALE, W. Manutenção de folhas ativas em bananeira'Nanicão' por meio do manejo das adubações nitrogenada e potássica e da irrigação. Revista Brasileira de Fruticultura, Jaboticabal, v.23, n.3, p.699-703, 2001. http://dx.doi.org/10.1590/S0100$\underline{29452001000300051}$

VILLAS BOAS, R.L.; FERNANDES, D.M.; BOARETTO, A.E.; GODOY, L.G. Fertirrigação: uso e manejo. In: CONGRESSO BRASILEIRO DE CIÊNCIA DO SOLO, 30., 2005, Recife. Anais [...]. SBCS, 2005. 